\title{
Determination of Meteoroid Orbits and Spatial Fluxes by Using High-Resolution All-Sky CCD Cameras
}

\author{
Josep M. Trigo-Rodriguez · José M. Madiedo $\cdot$ Peter S. Gural • \\ Alberto J. Castro-Tirado · Jordi Llorca · Juan Fabregat · Standa Vítek • \\ Pep Pujols
}

Received: 13 September 2007/ Accepted: 3 December 2007

(C) Springer Science+Business Media B.V. 2008

\begin{abstract}
By using high-resolution, low-scan-rate, all-sky CCD cameras, the SPanish Meteor Network (SPMN) is currently monitoring meteor and fireball activity on a year round basis. Here are presented just a sampling of the accurate trajectory, radiant and orbital data obtained for meteors imaged simultaneously from two SPMN stations during the continuous 2006-2007 coverage of meteor and fireball monitoring. Typical astrometric uncertainty is 1-2 arc min, while velocity determination errors are of the order of $0.1-0.5 \mathrm{~km} / \mathrm{s}$, which is dependent on the distance of each event to the station and its
\end{abstract}

\footnotetext{
J. M. Trigo-Rodriguez ( $\bowtie)$

Institute of Space Sciences (CSIC), Campus UAB, Facultat de Ciències, Torre C5-parell-2 $2^{\mathrm{a}}, 08193$ Bellaterra, Barcelona, Spain

e-mail: trigo@ieec.uab.es

J. M. Trigo-Rodriguez $\cdot$ J. Llorca

Institut d'Estudis Espacials de Catalunya (IEEC), Edif. Nexus, c/Gran Capità, 2-4, 08034 Barcelona, Spain

J. M. Madiedo

Facultad de Ciencias, Universidad de Huelva, Huelva, Spain

P. S. Gural

Science Applications International Corp, 14668 Lee Road, Chantilly, VA 20151, USA

A. J. Castro-Tirado $\cdot$ S. Vítek

Instituto de Astrofísica de Andalucía (IAA-CSIC), Camino Bajo de Huétor 50, 18008 Granada, Spain

J. Llorca

Institut de Tècniques Energètiques, Universitat Politècnica de Catalunya, Diagonal 647, ed. ETSEIB, 08028 Barcelona, Spain

J. Fabregat

Observatori Astronòmic, Universitat de València, Paterna, València, Spain

P. Pujols

Grup d'Estudis Astronòmics (GEA) and Agrupació Astronòmica d'Osona, Barcelona, Spain
} 
particular viewing geometry. The cameras have demonstrated excellent performance for detecting meteor outbursts. The recent development of automatic detection software is also providing real-time information on the global meteor activity. Finally, some examples of the all-sky CCD cameras applications for detecting unexpected meteor activity are given.

Keywords Meteors · Meteoroids · Meteoroid stream · All-sky camera · CCD camera $\cdot$ Astrometry $\cdot$ Heliocentric orbits

\section{Introduction}

Trigo-Rodríguez et al. (2004) had reported on the first steps in the deployment of lowscan-rate, all-sky CCD cameras that operate up to +2 to +3 limiting magnitude for meteors. A general overview of observations recorded in 2006, including the main meteor activity highlights and a list of bright fireballs, has been published in previous papers (Trigo-Rodríguez et al. 2006a, b, 2007). The establishment of the SPanish Meteor Network (SPMN) was keyed off the development and deployment of innovative low-scan-rate, allsky CCD cameras (Trigo-Rodríguez et al. 2004a). Presented herein are the trajectory and orbital results obtained with those systems during the years 2006 through 2007, that had undergone an equipment design upgrade that incorporated internal devices for accurately measuring meteor velocities. Results to be shown were obtained by the newer all-sky CCD stations set up in Catalonia during 2006. In particular, that year was excellent for our network due to favourable weather (generally dry with extraordinary clear weather conditions during autumn and winter) that guaranteed almost a continuous record of meteor activity.

We provide examples of trajectory and orbital results of meteors in the $[-10,+3]$ magnitude range, with limiting magnitudes substantially fainter than conventional all-sky photographic cameras (see e.g. review by Spurný et al. 2007). These data were obtained by using the low-scan rate, all-sky CCD cameras operated in Catalonia (Table 1). The reason for this selection is because the Catalan stations were built with internal rotating shutters to get accurate measurements of meteor velocities (Trigo-Rodríguez et al. 2007). We will focus here on some trajectory and orbital examples obtained by using our all-sky CCD cameras. In a parallel paper we also present video data obtained from three complementary video stations developed in Andalusia (Madiedo and Trigo-Rodríguez 2007).

Table 1 Stations of the SPMN involved in this work

\begin{tabular}{llllll}
\hline $\begin{array}{l}\text { Station } \\
\#\end{array}$ & Station (Province) & $\begin{array}{l}\text { Longitude } \\
(\mathrm{E})\end{array}$ & $\begin{array}{l}\text { Latitude } \\
(\mathrm{N})\end{array}$ & \multicolumn{1}{l}{$\begin{array}{l}\text { Alt. } \\
(\mathrm{m})\end{array}$} & $\begin{array}{l}\text { Imaging } \\
\text { system }\end{array}$ \\
\hline 1 & Montsec, OAdM (Lleida) & $00^{\circ} 43^{\prime} 46^{\prime \prime}$ & $42^{\circ} 03^{\prime} 05^{\prime \prime}$ & 1570 & AS \\
2 & Montseny (Girona) & $02^{\circ} 31^{\prime} 14^{\prime \prime}$ & $41^{\circ} 43^{\prime} 17^{\prime \prime}$ & 300 & AS \\
3 & Folgueroles (Barcelona) & $02^{\circ} 19^{\prime} 33^{\prime \prime}$ & $41^{\circ} 56^{\prime} 31^{\prime \prime}$ & 580 & WF \\
\hline
\end{tabular}

Acronyms for the different imaging systems are: AS (low-scan-rate CCD all-sky camera), and WF (lowscan-rate CCD wide-field camera) 


\section{Instrumentation, Data Reduction and Observation Sites}

A description of the low-scan-rate, CCD cameras was published previously in TrigoRodríguez et al. (2004). The cameras are operated in a sequential imaging mode, each making 90 second exposures followed by a typical readout time of 30 or $15 \mathrm{~s}$, depending on whether the readout is controlled by a parallel or USB port respectively. Currently, the CCD imagery is analyzed using a set of image processing modules that have recently been developed. The initial stage consists of obtaining a frame difference among two sequential images, with the result that stars appear as continuous or trailed tracks (non-guided images) characterized by a positive streak followed by an equal length negative streak (Fig. 1a). The background, in the periphery of the all-sky view where obstacles such as buildings and trees are visible, is effectively removed by the frame differencing. Since our subtraction occurs on the raw images without any image transformations, each differenced element from the two images has the same pixel response and the background mean is effectively eliminated without the need for flat fielding the frames. The residual variance will depend on the response of each pixel and ideally should be flat fielded after the difference is taken, but since we do not have a flat field available, this step is ignored and can be justified because of the spatial culling in the next step. A threshold is estimated (mean $\pm 2 \times$ standard deviation) for a local region around each pixel exceedance with high and low outliers removed. Thus any pixel that possesses a large variance due to either hot or cold pixel response, is eliminated from the threshold estimation. For the algorithm to operate successfully without flat fielding, the majority of pixels must have nearly equivalent responses across the focal plane with only a few hot or cold pixels in existence. This is typically a good assumption for most CCD sensors.

Using the above mentioned procedure, meteor detection consists of peak finding after a spatial re-mapping of pixel exceedances above or below the local thresholds through the use of the Hough Transform (HT) (see e.g., Hough 1962; Duda and Hart 1972). Using a

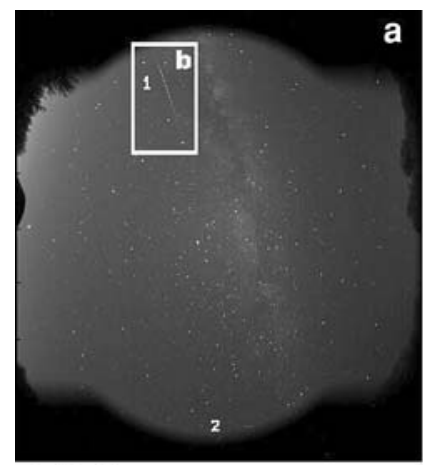

Initial image

Raw image pair $\Rightarrow$ differenced
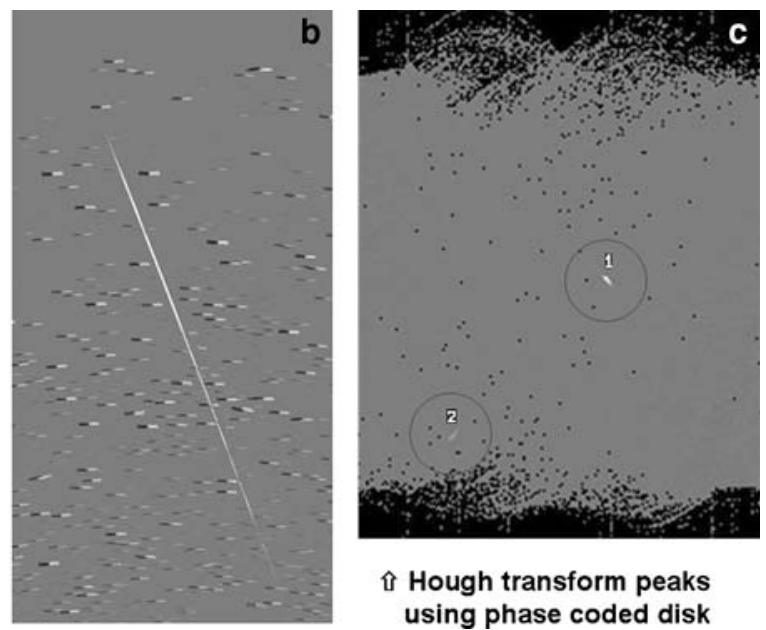

Fig. 1 Meteor detection steps. Plot (a) shows a representative all-sky image with stars, background, and two meteors (marked as 1 and 2). Plot (b) shows the difference frame where star trails have equal parts positive (white) and negative (black) components whereas meteors are predominantly only one colour. Plot (c) is the Hough space output showing two peaks at the angle and offset of the lines aligned with the meteor (for more details see Gural 2007) 
modification to the traditional HT, we apply a phase-coded disk to each pixel exceedance to estimate the local orientation of the track. A phase coded disk (see Clode et al. 2004) is a convolution kernel that when applied to a binary image, determines the orientation angle of a line within a local region encompassed by the spatial extent of the kernel. Given the position of an exceedance pixel and angle of the line through that pixel, only a single cell in the Hough transform space is incremented rather than a hypothesized set of all angles through that pixel in traditional HT processing. This helps avoid the generation of Hough self noise (the "butterfly" pattern) and its associated reduced probability of detection.

When applying the HT to the SPMN imagery, star trails of both unguided exposures are mutually cancelling in the Hough space accumulator (Fig. 1b) since they have equal positive and negative contributions after the frame difference and nearly the same position and orientation angle. Additionally, a mechanism of false alarm mitigation is implemented that excludes regions in Hough space for every line detection found (Fig. 1c) thus helping to avoid multiple detections of the same meteor. This assumes that two meteors are unlikely in the same location and direction, which does not affect the final goal, that is, saving the image for future visual inspection/confirmation. Images with positive identification are saved and can be later re-examined in order to confirm the identification of the suspected streak as a meteor, and also to look for additional tracks by visual inspection. More details on the software are given in Gural (2007).

Meteor photometry of the CCD images is a field that we currently have under study. CCD cameras allow an analyst to estimate stellar and meteor magnitudes very accurately since they provide photon counts for every pixel. In all-sky CCD imaging we adopt an approach where meteor magnitudes are derived by comparing the intensity level of the pixels close to the maximum luminosity of the meteor trail and nearby stars. Taken into account is the different angular velocity of the meteors as a function of the distance to the radiant, and the typical duration of flares. An example of a photometric curve is given in Fig. 2. Additional extinction corrections are also considered to get the absolute magnitude of meteors that appear below $30^{\circ}$ of altitude.

Positional measurements of the meteor track from the CCD images are currently performed by using the Maxim DL software package (http://www.cyanogen.com/). Accurate astrometry of medium sized field-of-view images is obtained for both stars and meteors by using the polynomic astrometric method published by Steyaert (1990). However, all-sky images have a more complex astrometric reduction to account for the image distortion of wide-eye lenses (Ceplecha 1987; Borovička et al. 1995). Both methods are currently implemented in our Amalthea software (Madiedo and Trigo-Rodríguez 2007) that has been written in C and follows the general numerical procedures described by Press et al. (1992). The new Amalthea software has wider applicability that our previous Network software (Trigo-Rodríguez et al. 2002, 2004b). With the present angular resolution ( $\sim 1$ arc minute) of the camera systems, the equatorial coordinates of the meteors are computed with an astrometric accuracy of approximately $0.01^{\circ}$, which also determines the apparent and geocentric radiant of any meteor. Reconstruction of the atmospheric trajectory and radiant is performed by using the method of intersecting planes developed by Ceplecha (1987). From the astrometric measurements of the shutter breaks along the trajectory and chopping rate, the velocity of the meteoroid is derived. The velocity measured for each shutter-break was obtained as well as the preatmospheric velocity $V_{\infty}$ from the velocity measured in the earliest portion of each meteor trajectory (usually in the first 3 or 5 breaks, when deceleration is weak). At present we do not apply deceleration corrections to the measured velocity, but we plan to apply a dynamic model of the meteor flight in the future by using Amalthea software. Finally, in order to determine orbital elements from our trajectory data 

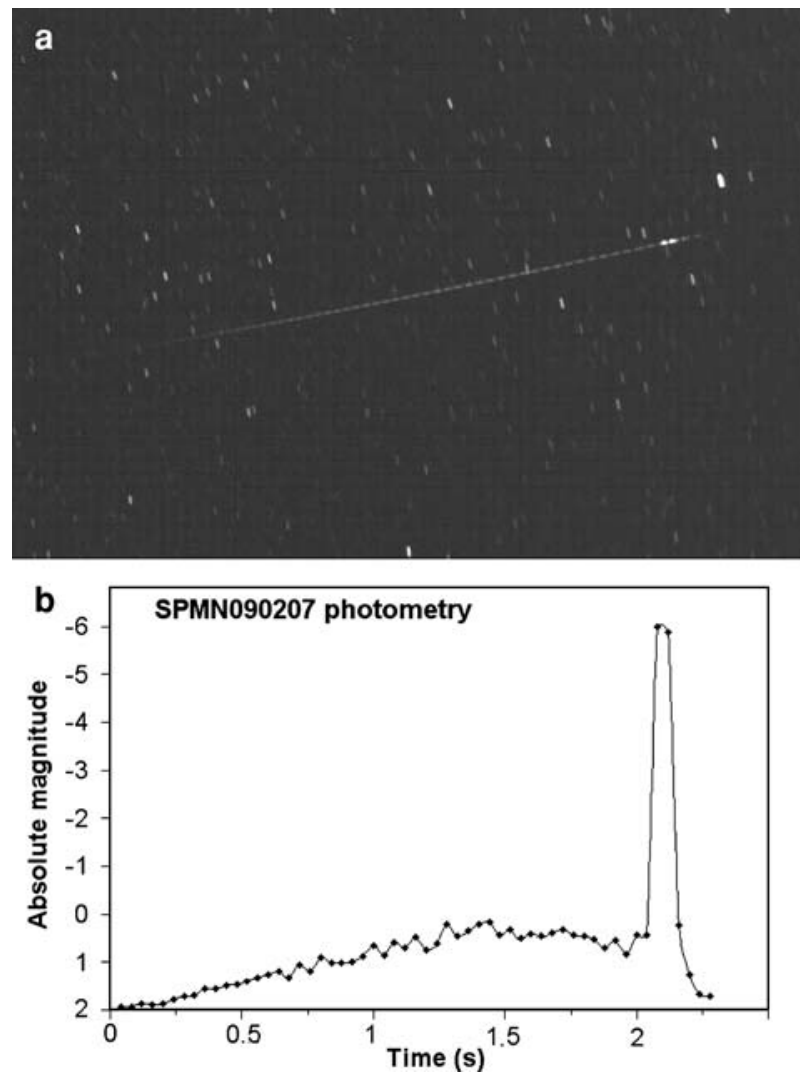

Fig. 2 Photometry of the SPMN090207 "Tordera" fireball. Each point represents the integrated signal computed for each different shutter break, once the background has is removed for considering only the light emitted by the fireball. (a) Image taken from Montseny all-sky camera, and (b) Absolute visual magnitude plotted as function of time. For star identification, Castor is the brightest star in the field, nearby the fireball's main flare

we used the MORB program provided by Ceplecha et al. (2000) from the Ondrejov Observatory in the Czech Republic.

\section{Observations: Spatial Fluxes, Trajectory, Radiant and Orbital Data}

Continuous monitoring of meteor activity using our all-sky CCD cameras allows for the determination of meteoroid spatial fluxes for minor showers. An example of unexpected activity identified from all-sky CCD imaging is the 2006 Orionid outburst that we quickly reported to the community (Trigo-Rodríguez et al. 2006, 2007b). By estimating the meteor magnitudes from the images, we obtained a magnitude distribution for the night of Oct. 20-21, 2006 (Trigo-Rodríguez et al. 2007a). From the derived population index ( $r=1.4 \pm 0.4, N=33$ ) we determined an incident flux of 415 meteoroids brighter than magnitude +6.5 per $\mathrm{km}^{2}$ using a high fidelity meteor simulation (Gural and Jenniskens 2000; Molau et al. 2002). The meteor simulation determines the incoming spatial flux by 
modelling the camera's sensitivity characteristics, the geometric loss terms, the change in radiant position, the meteoroid stream's particle distribution, and associated entry parameters, to determine the meteoroid incident density that is required to match the actual counts seen by the sensor's field of view and look orientation. Using this flux value and the same simulation tool, but with human visual perception parameters and loss models substituted (Gural 2004), we estimated a visual (human) ZHR $=50 \pm 15$ (TrigoRodríguez et al. 2007). In order to compute the spatial flux of meteoroids producing meteors brighter than +6.5 per $\mathrm{km}^{2}$, as well as an equivalent visual Zenithal Hourly Rate (ZHR), the count rates must be obtained from the all-sky systems for a consistent limiting magnitude of detection.

Multiple-station detections of meteors, as opposed to single station observations, allows the computation of accurate trajectory and orbital data when imaged by all-sky CCD cameras. Table 2 shows the absolute magnitude $\left(M_{\mathrm{v}}\right)$ in the visual range, the height for beginning, maximum and terminal light $\left(H_{\mathrm{b}}, H_{\mathrm{max}}, H_{\mathrm{end}}\right)$, the geocentric radiant $\left(\alpha_{\mathrm{g}}, \delta_{\mathrm{g}}\right.$ to Eq. 2000.00), the convergence angle $(Q)$ of the meteor seen from both stations and the infinity, geocentric, and heliocentric velocities $\left(V_{\infty}, V_{\mathrm{g}}, V_{\mathrm{h}}\right)$. Note that only those meteors with $Q>20^{\circ}$ were considered. From the radiant position, apparition time, and velocities estimated for the meteors listed in Table 2, the orbital elements were derived and shown in Table 3.

\section{Discussion}

Determination of spatial number densities for meteor showers in near real time from all-sky CCD imagery is considered a significant advance in the meteor community. We have already applied the regional coverage of the SPMN cameras to get quick reports of unusual meteor activity, as e.g. those reported for the 2006 Orionid outburst (TrigoRodríguez et al. 2006a) or the Kappa Cygnid fireball display (Trigo-Rodríguez et al. 2007c). We also reported that there was no evidence of background meteor activity from the dust trail of comet C/1911 N1 (Kiess) a few hours before the peak reported from United States (Jenniskens et al. 2007).

Direct astrometry on CCD images can provide accurate astrometry of meteors. When they are observed from several stations, trajectory and orbital data can be derived in just a few hours. The observational uncertainties in trajectory data and orbital elements reported in Tables 2 and 3 are similar to those reported by small photographic camera networks but they are still less accurate than those obtained by European Network (EN) photographic cameras (see e.g. Spurný et al. 2004). The main reason for this is the order of magnitude better spatial resolution of the EN all-sky photographic cameras, as was already pointed out in our previous work (Trigo-Rodríguez et al. 2004). In spite of this, some of the presented orbital data are perfectly comparable because the geometry of the meteor's appearance also plays an important role. On the other hand, developing techniques in higher resolution pixel interpolation (e.g. Quine et al. 2007) can be useful for reducing astrometric uncertainties.

The data shown in Tables 2 and 3 demonstrate that all-sky CCD cameras can collect valuable trajectory and orbital information on meteors. The quick reduction of data has been demonstrated to be important for alerting our community of unusual meteor activity on short time-scales (Trigo-Rodríguez et al. 2006, 2007b, c). Although the number of trajectories and orbital results presented herein has been limited for brevity, we think that the sample is well representative of meteors spanning different velocities, magnitudes, and 

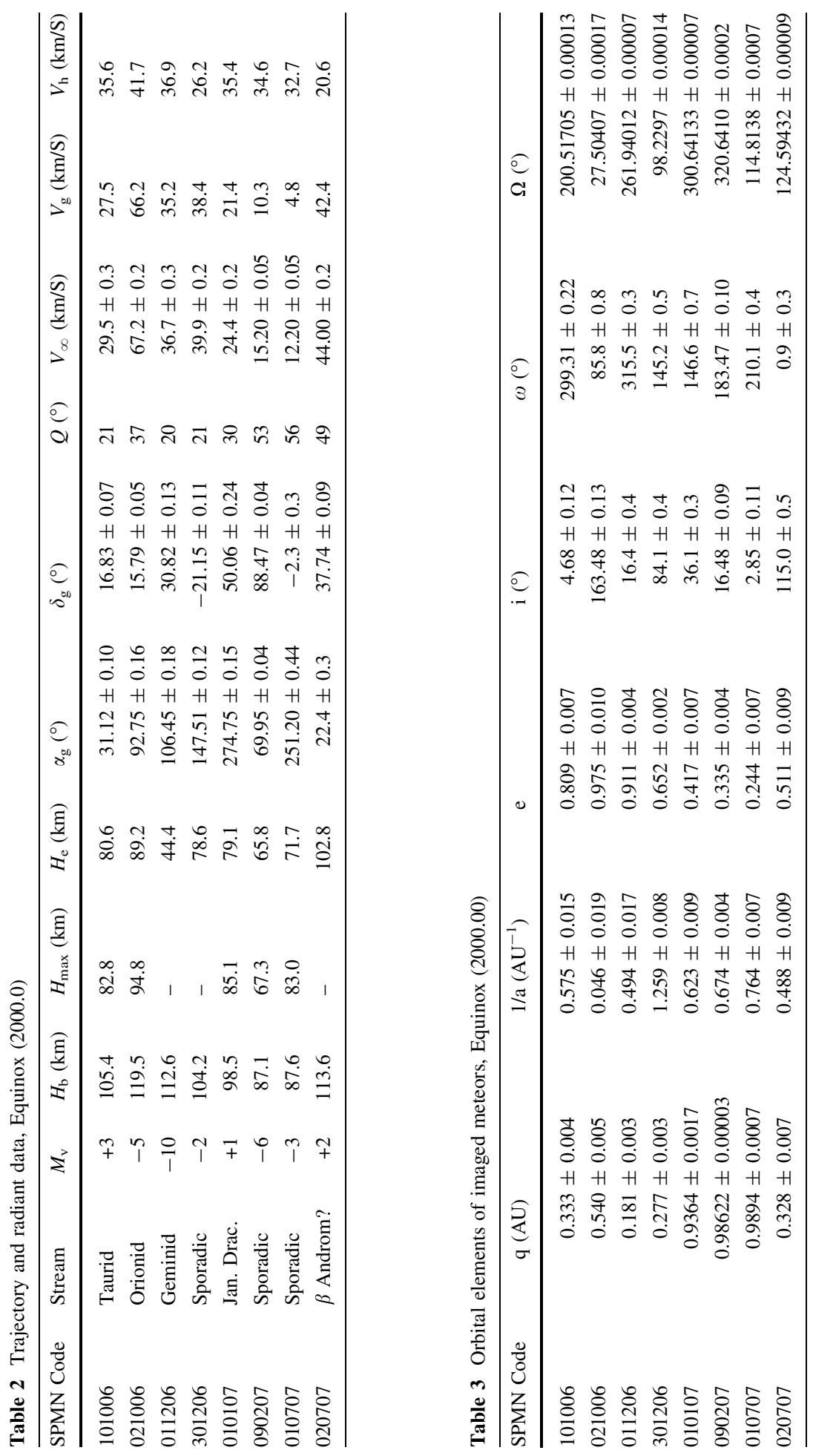

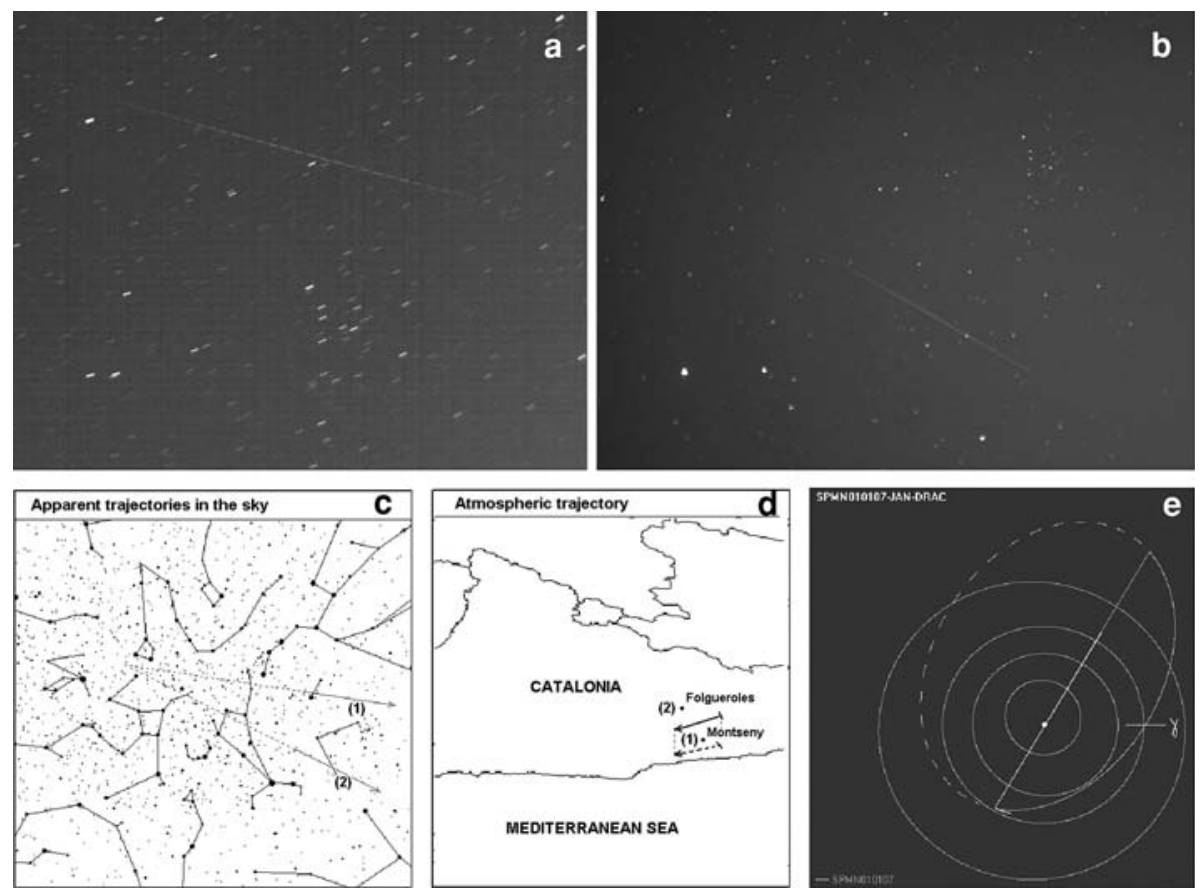

Fig. 3 Part of the all-sky CCD image centred in the +1 magnitude January Draconid (SPMN010107) recorded from (a) Station (1) Montseny with a shutter providing 25 breaks/s, and (b) Station (2) Folgueroles (c) Apparent trajectory of the meteor from both stations. (d) Atmospheric trajectory and its projection on the ground. (e) The heliocentric orbit of the meteoroid projected on the ecliptic plane, where the orbits of the terrestrial planets are shown for comparison

properties from major showers, minor showers, and sporadics. Continuous monitoring also permits searching for and studying poorly observed minor showers. A good example is a +1 magnitude meteor presented in Fig. 3 (labelled as SPMN010107 in Table 2). Initially identified as a sporadic when compared to orbital elements obtained by Sekanina (1976), we later found similarity with a stream identified by Kronk (1988) as the January Draconids. Many of the meteors contained in Tables 2 and 3 exhibited magnitudes well below the typical limit of conventional (photographic) all-sky cameras. At the bright bolide extreme, astrometry has been found to be feasible since saturation of the imaging chip usually occurs over just a small portion of the fireball path. By way of example we included the orbit of a very bright Geminid bolide (SPMN011206) recorded during the extraordinary 2006 Geminid display (Fig. 9 of Trigo-Rodríguez et al. 2007), and the orbit of a -6 sporadic event (SPMN 090207).

\section{Conclusions}

Several examples were presented of the capability of the recently developed all-sky CCD camera systems described in Trigo-Rodríguez et al. (2004) for both trajectory and orbital determination of meteors. The deployment of complementary detection systems (video, forward scatter, infrasound, etc...) in a wide area network, are our future goals for 
achieving multi-instrument studies like those previously reported (Spurný et al. 2004; Weryk et al. 2007). At the current stage of development for the SPMN, the main conclusions of this paper are:

(a) Low-scan-rate, all-sky CCD cameras applied to meteor monitoring (Trigo-Rodríguez et al. 2004) can provide very valuable information on spatial fluxes of meteor showers. Detection of unexpected meteor activity is feasible due to the better sensitivity of the current CCD cameras over previous all-sky systems reaching meteor limiting magnitudes of +2 to +3 .

(b) Reliable trajectory, radiant and orbital data of the imaged meteors can be computed on shorter time-scales (a few hours) than other existing processing systems with exception of real-time video systems.

(c) Subsequent advances in automatic meteor detection, stream association, data analysis, and other computational reduction tasks will provide important progress in improving our real-time alarm capabilities. All this will contribute to an increase in the amount of data available on poorly studied meteor showers that would be very valuable to the meteor community.

Acknowledgements These results were achieved by using wide-field automatic digital cameras described in the Spanish patent application number 200501127, filed May 2005, and later continued in the PCT document number PCT/ES06/070057. The 2006 development of the internal mechanism for obtaining meteor velocities by J.M.T-R is under consideration for a patent. The authors would like to thank Instituto Nacional de Técnica Aeroespacial (INTA) and Consejo Superior de Investigaciones Cientificas (CSIC) for the development of the all-sky CCD camera prototype in 2002. Finally, J.M.T.-R thanks Ministerio de Educación y Ciencia (MEC) for a JdC grant.

\section{References}

J. Borovička, P. Spurný, J. Keclikova, A new positional astrometric method for all-sky cameras. Astron. Astrophys. Suppl. Ser. 112, 173-178 (1995)

Z. Ceplecha, Geometric, dynamic, orbital and photometric data on meteoroids from photographic fireball networks. Bull. Astron. Inst. Czechosl. 38, 222-234 (1987)

Z. Ceplecha, P. Spurny, J. Borovička, MORB software to determine meteoroid orbits. (Ondrejov Observatory, Czech Republic 2000)

S. Clode, E. Zelniker, P. Kootsookos, V. Clarkson, A phase coded disk approach to thick curvilinear line detection. In 2004 XII. European Signal Processing Conference, Vienna, Austria, September 2004 (2004) pp. 1147-1150

R.O. Duda, P.E. Hart, Use of the Hough transformation to detect lines and curves in pictures. Comm. ACM 15(1), 11-15 (1972)

P. Gural, P. Jenniskens, Leonid storm flux analysis from one Leonid MAC video AL50R. Earth Moon Planet. 82/83, 221-247 (2000)

P. Gural, A human visual perception model and its impact on population index estimation, ZHR, and Best Look Direction. WGN, J. IMO 32(4), 97-108 (2004)

P. Gural, Algorithms and software for meteor detection. Earth Moon Planet. (2007) doi:10.1007/ s11038-007-9161-7

P.V.C. Hough, Methods and means for recognizing complex patterns. U.S. Patent 3,069,654 (1962)

Jenniskens et al., 2007 Aurigid Meteors. Central Bureau Astronomical Telegram, CBAT \#1045, IAU (2007)

G.W. Kronk, Meteor showers: A descriptive catalogue. (Enslow Publ., Hillside, USA, 1988)

A.B.C. Lovell, Meteor Astronomy (OUP, Oxford, 1954)

J.M. Madiedo, J.M. Trigo-Rodríguez, Multi-station video orbits of minor meteor showers. Earth Moon Planet. (2007). doi:10.1007/s11038-007-9215-x

S. Molau, P. Gural, O. Okamura, Comparison of the American and the Asian 2001 Leonid meteor store. WGN J. Int. Meteor Org. 30-1, 3-21 (2002)

W.H. Press, S.A. Teukolsky, W.T. Vetterling, B.P. Flannery, Numerical Recipes in C (Cambridge University Press, NY, 1992) 
B.M. Quine, V. Tarasyuk, H. Mebrahtu, R. Hornsey, Determining star-image location: a new sub-pixel interpolation technique to process image centroids. Computer Phys. Comm. 177, 700-706 (2007)

Z. Sekanina, Statistical model of meteor streams IV. A study of radio streams from the synoptic year. Icarus 27, 265-321 (1976)

P. Spurný, J. Borovička, P. Koten, Multi-instrument observations of bright meteors in the Czech Republic. Earth Moon Planet. 95, 569-578 (2004)

P. Spurný, J. Borovička, L. Shrbeny, Automation of the Czech part of the European fireball network: equipment, methods and first results. in Near Earth Objects, our Celestial Neighbors: Opportunity and Risk, ed. by G.B. Valsecchi and D. Vokrouhlicky, Cambridge Univ. Press. Proceedings IAU Symposium No. 236, 121-130 (2007)

C. Steyaert, Photographic Astrometry (Edited by the International Meteor Organization, Belgium, 1990)

J.M. Trigo-Rodríguez, J. Llorca, J. Fabregat, On the origin of the 1999 Leonid storm as deduced from photographic observations. Earth Moon Planet. 91, 107 (2002)

J.M. Trigo-Rodríguez, A.J. Castro-Tirado, J. Llorca, J. Fabregat, V.J. Martínez, V. Reglero, M. Jelínek, P. Kubánek, T. Mateo, A. de Ugarte Postigo, The development of the Spanish Fireball Network using a new all-sky CCD system. Earth Moon Planet. 95, 553 (2004a)

J.M. Trigo-Rodríguez, J. Llorca, E. Lyytinen, J.L. Ortiz, A. Sánchez Caso, C. Pineda, S. Torrell, 2002 Leonid storm fluxes and related orbital elements. Icarus 171, 219 (2004b)

J.M. Trigo-Rodríguez et al., Orionid Meteors. Central Bureau Astronomical Telegram, CBAT \#698, IAU (2006a)

J.M. Trigo-Rodríguez, J. Llorca, A.J. Castro-Tirado, J.L. Ortiz, J.A. Docobo, J. Fabregat, The Spanish Fireball Network. Astron. Geophys. 47, 6-26 (2006b)

J.M. Trigo-Rodríguez, J.M. Madiedo, A.J. Castro-Tirado, J.L. Ortiz, J. Llorca, J. Fabregat, S. Vítek, P.S. Gural, B. Troughton, P. Pujols, F. Gálvez, Spanish Meteor Network: 2006 continuous monitoring results. WGN J. IMO 35(1), 13-22 (2007a)

J.M. Trigo-Rodríguez, J.M. Madiedo, J. Llorca, P.S. Gural, P. Pujols, T. Tezel, The 2006 Orionid outburst imaged by all-sky CCD cameras from Spain: meteoroid spatial fluxes and orbital elements. Monthly Notices Roy. Astron. Soc. 380, 126-132 (2007b)

Trigo-Rodríguez J.M. et al., Kappa Cignids 2007. Central Bureau Astronomical Telegram, CBAT \#1055, IAU $(2007 \mathrm{c})$

R.T. Weryk, P.G. Brown, A. Domokos, W. Edwards, Z. Krzeminski, S.H. Nudds, D.L. Welch, The Southern Ontario all-sky meteor camera network. Earth Moon Planet. (2007). doi:10.1007/s11038-007-9183-1 